\title{
Scuola di Economia dello Sviluppo Integrale: I.P.I. contro P.I.L.
}

\author{
Prof. Romeo Ciminello*
}

\begin{abstract}
There are three levels of human development: social project, political project, economic project to reach " integral human development ". Lonergan theory: value is in the man self-conscious and humanly auto-proportioned. Value is not economic, is human and assumes the economy develops not already and on its own autonomous laws, but thanks to practice application of the ideas of man and his intelligence. Productivity system: " point-to-point, point-to-line and point-to- surface": no limit to the creativity of human being. Without human cooperation no ideas of economic development " because he dominates economic mechanisms through his intelligence his selfappropriation: his consciousness of being the subject. Good of order leads to integral human development, since economics becomes a human science related to political economy to achieve better conditions of standards of living for the whole of humanity. The aim: creation of a renewed "School of development economics", based on the good of order for the integral development of all countries, different from last century ideas, because development is integrative, transparent and complete, refusing economic liberalism, and defined the "new name for peace" . Economy is a fact of life, is knowledge, so no more mere transfer of wealth: development comes from transfer of knowledge. Its vector characters : balanced, sustainable and durable. Let's leave GDP as measure of country development for U.P.I. (Unexploited Potential Index) in its human characters, animals and environment in which investment has to supply a planned social return.
\end{abstract}

\section{Introduzione}

La visione grandemente attuale e più perspicace di B.J. Lonergan nelle scienze sociali, appare quella dell'economia ${ }^{1}$ relativa allo sviluppo, la cui interpretazione così avanzata già per il suo tempo, tanto che gli economisti di allora non riuscirono a comprendere, resta ancora oggi per la maggior parte di quelli esistenti, piuttosto difficile da applicare.

Il suo saggio Essay on Circulation Analysis ${ }^{2}$ infatti presenta un pensiero così lungimirante che anche i curatori dell'opera hanno certamente faticato non poco per presentarlo in maniera oserei dire ortodossa e fedele alla sua rivoluzionaria visione .

Ciò si evidenzia dal fatto che i curatori nel riportare il suo pensiero e la sua formazione paragonano la sua visione economica a quella dei grandi economisti come Smith, Marx,

${ }^{1}$ B.J. Lonergan For a New Political Economy, ed. By Ph.J. McShane (CWBL 21), University of Toronto Press, Toronto 1998 - Traduz. Ital. di Michele Tomasi Studi di Economia Città Nuova Editrice, Roma Novembre 2013

2 B.J. Lonergan Macroeconomics Dynamics: An essay on Circulation Analysis (Collected Works of Bernard Lonergan, Volume 15). Edited by F.G. Lawrence, P. H. Byrne, and C.C. Hefling, Jr.. Toronto: University of Toronto Press, 1999. 
Marshall, Keynes, Kalecky e Schumpeter ${ }^{3}$ in un confronto però solo a livello ideologico, senza tener conto dell'origine delle sue posizioni, dimostrando così di non esser stati in grado di percepire e giustamente esprimere la grande novità del suo pensiero, determinata dalla visione socio-economica smisuratamente distante da tutti gli economisti citati.

Lonergan ancorché costretto ad utilizzare termini e concetti adottati da tutti gli economisti, non aveva ancora né termini di paragone e né metri di misura per esprimere quei contenuti insiti nei nuovi concetti da lui individuati e che nessuno dei suoi contemporanei aveva ancora minimamente accennato, pur se anch'essi chiamavano l'economia "politica" in termini micro e "politica economica" in termini macro.

Ciò che oggi gli studiosi scoprono dal suo contributo è la sua grande intuizione che permette al pensiero economico contemporaneo di non smarrirsi: i ritmi della realtà dei tre livelli di progettualità dello sviluppo umano, vale a dire la progettualità sociale, come elemento fondativo della pace nella comunità degli uomini, la progettualità politica mirata alla costruzione del progetto sociale per far convergere l'azione verso il bene comune ed infine la progettualità economica quale elemento di raccordo e supporto delle prime due per l'impiego del patrimonio disponibile come potenzialità esistenti in termini umani, animali e ambientali. Il tutto volto allo "Sviluppo integrale dell'uomo" come presentato dall'enciclica Populorum Progressio. ${ }^{4}$

In tale contesto seguiremo un percorso metodologico che partendo dai dati di fatto si indirizza verso criteri di giudizio che coinvolgono la razionalità e la responsabilità che il pensiero economico deve assumere, cercando di giungere infine alla fondazione di una conoscenza che transiti per la bellezza della conoscenza e l'amore verso le soluzioni più vicine al riconoscimento della dignità umana che si misura dal grado di libertà e di creatività in termini lavorativi di ciascun essere umano.

Le tappe del percorso anche se schematizzate e non passibili di accurato approfondimento saranno comunque presentate sotto il profilo scientifico come punti di riferimento sui quali costruire e verificare un pensiero che andrà a rivoluzionare l'impostazione teorica delle formulazioni economiche attualmente in essere.

La prima cosa che verrà affrontata sono le ragioni che spingono verso una nuova teoria economica dello sviluppo a cui seguirà la visione di Lonergan sull'economia in termini di assunto teorico. Nel prosieguo si affronta il problema del sistema produttivo, quale base ineludibile di qualsiasi indice economico di sviluppo che fondandosi nell'argomento successivo dell'impresa come bene d'ordine permette di capire come si giunge all'idea di una scuola di pensiero per una rinnovata economia dello sviluppo che abbia come finalità la pace e come mezzo la conoscenza da cui si dipartono i vettori di sviluppo che saranno raggruppati in un unico aggregato capace di fare chiarezza sul significato di sviluppo perché sostituisce nella funzione l'attuale indice PIL con quello dell'IPI.

La conclusione infine non sarà chiusa da assunti definitivi, ma resterà auspicabilmente aperta al contributo di tutti gli studiosi che credono nelle potenzialità dell'essere umano ed alle sue capacità di creare un mondo dove lo sviluppo possa essere veramente equo, sostenibile e durevole.

${ }^{3}$ Cfr. ed ital. cit., Introduzione pp. 20-27.

4 Palo VI, Enciclica Populorum Progressio nn. 14 e 15. 


\section{Le ragioni di una nuova Economia dello Sviluppo}

Il problema della visione economica secondo Lonergan è di natura assiologica perché riscontra la incapacità dell'uomo di determinare la fonte del valore e di organizzarne il percorso logico all'interno dell'azione economica. Infatti l'analisi contrastata e sofferta del pensiero dei marginalisti e degli economisti della scuola austriaca gli permise di intravedere uno spiraglio nuovo nel concetto di valore. Egli infatti fonda la sua visione delle ragioni che invocano una nuova economia dello sviluppo muovendo una critica molto forte alle concezioni su cui si sono sviluppate nella storia le due diverse visioni economiche, mettendo in evidenza che si possono rilevare due diversi tipi di visione, il primo che possiamo chiamare "la vecchia economia politica" e il secondo "la nuova scienza economica". Le critiche rivolte alla prima riguardano il fatto che nella sua evoluzione storica "la vecchia economia è stata riconosciuta colpevole di molti errori, dimostrati dall'esperienza dei fatti; errori di metodo e di principio." 5 Gli errori fatti, andavano corretti, ma asserisce Lonergan, lo furono in maniera sbagliata perché pur presentando un grado di accuratezza in termini scientifici, l'analisi economica mancava in realtà dello spirito democratico. Tale economia politica infatti era "Incapace di riferirsi in modo efficace alla gente comune, essa non può che mettere in dubbio la capacità dei capi democratici di seguire il corso tortuoso del suo pensiero. Il risultato è che l'unica soluzione che essa può sembrare offrire ai problemi economici è quella di fornire un gruppo di esperti a una incipiente burocrazia o di fornire dei tecnici ad uno Stato totalitario." "La sua critica invece riguardo alla nuova scienza economica, che si è imposta via via nel corso del secolo passato, è che "Non possiamo contare sulla nuova scienza economica: è accurata, ma può risolvere i problemi reali soltanto eliminando la democrazia" Ecco perché conclude con una affermazione che indica chiaramente la necessità di fare un importante salto di paradigma riguardo alla visione economica: "ciò di cui abbiamo bisogno è una nuova economia politica, che sia libera dagli errori della vecchia, un'economia democratica che possa produrre consigli pratici per uomini comuni." 8 Egli spiega come ci sia bisogno di una nuova economia più attenta e mirata alla soluzione dei problemi economici della gente comune perché: "la scienza economica non ha corretto l'economia politica muovendo verso il campo più generale, ed effettuando così le correzioni senza perdere lo spirito democratico del vecchio movimento, ma piuttosto rimanendo allo stesso livello di generalizzazione e guadagnando il terreno perduto entrando nel campo più particolareggiato della statistica, della storia e di una e più raffinata analisi delle motivazioni psicologiche e dell'integrazione delle decisioni di scambio." Ovviamente in questo ragionamento egli si riferisce alla maniera con cui Schumpeter articola il suo studio, nella sua visione sociologica dell'economia unitamente alla sua analisi metodologica derivanti dalle concezioni, oggi molto discutibili, promosse dalla Scuola Austriaca.

\footnotetext{
${ }^{5}$ B.J. Lonergan, op. cit., pag. 35

6 ibidem

7 Ibidem; democrazia intesacome libertà di partecipazione

8 ibidem

9 B.J. Lonergan, op. cit., pag. 38
} 
La convinzione di Lonergan, che mi sento di sottoscrivere in pieno è che "quanto più l'economia si sforza di essere una scienza esatta, tanto più essa diventa incapace di parlare agli uomini, e tanto maggiore è la sua necessità di trattare gli uomini come le scienze esatte trattano gli atomi e le cavie: deve sottoporli a condizioni di laboratorio ma con una GPU ${ }^{10}$ che ve li costringa e un gruppo di commissari per pianificare gli investimenti;.."11. Secondo Lonergan ciò che è necessario è una maggiore generalizzazione degli assunti economici perché "nella storia economica, le conclusioni generali dipendono molto più dalla validità dei principi generali di interpretazione che non dall'accuratezza dei dettagli concreti."12 Se finora quindi abbiamo inteso l'economia nel suo circuito di produzione di ricchezza, domanda, offerta e livelli di costo o di prezzo inquadrati in modelli sempre più precisi e di tipo econometrico soprattutto per dare un significato quantitativo alle variazioni intercorrenti nel conflitto tra capitale e lavoro e tra interesse e profitti nonché nella simmetricità di produzione, trasformazione, distribuzione e consumo, ebbene dobbiamo cambiare paradigma di riferimento. Dobbiamo partire da un punto di vista generale perché questo includendo il particolare fornisce una visione più completa del contesto. La sua teoria ancora tutta da scoprire si collega alla sua triplice concezione di valore come vero e falso, terminale e originante ed infine gerarchico. Questa interpretazione del valore attribuita alla sfera economica però riflette e caratterizza la teoria del valore in Lonergan che è l'attinenza di questa con l'uomo, anzi con l'uomo autocosciente ed umanamente autoproporzionato. Anche se per molti potrebbe sembrare una posizione ermeneutica di difficile comprensione in realtà approfondendo con attenzione il concetto si evince che il valore non è economico, bensì è umano ed assume una sua precisa peculiarità. La conclusione che se ne deriva è che l'economia si sviluppa non già per proprie leggi autonome, o "ferree" come le chiama, bensì grazie all'applicazione pratica delle idee dell'uomo e della sua intelligenza. Infatti il valore non dipende dalla terra, dalla rendita, o dal lavoro, ma certamente dall'innovazione di cui già Schumpeter si era fatto promotore. Egli configura in termini nuovi il concetto di produttività, generata da sistemi che egli definisce " punto a punto, punto su linea e punto sul piano” per indicare l'assenza di limiti alla creatività dell'uomo.

\section{Teoria di Lonergan}

La sua teoria del valore oltre ad essere legata strutturalmente alle idee dell'uomo è supportata anche da una visione dei cicli che le idee dell'uomo generano attraverso quello che egli verifica nella storia economica asserendo che " in ogni periodo della storia umana, dalle caverne preistoriche alle utopie che i nostri profeti descrivono con particolari tanto vividi, tra i primitivi raccoglitori di frutta, tra cacciatori e pescatori, all'alba prigiemia della civiltà agricola, lungo il Nilo d'Egitto e l'Eufrate di Babilonia, sotto il misticismo dell'India, la raffinatezza della Cina, il pensiero greco, la legge romana, attraverso il tumulto dell'età oscura e il fermento del periodo medioevale, nell'espansione europea e nel mondo moderno, ovunque si trova il flusso pulsante, la serie ritmica delle

\footnotetext{
10 Abbreviazione di Gousdarstvennoye Politischeskoye Upravleniye, I servizi statali e di sicurezza politica nell'Unione Sovietica.

${ }^{11}$ Ibidem

12 B.J. Lonergan, op. cit., pag. 41
} 
attività economiche dell'uomo"13 La sua percezione del processo mondiale e dell'interazione di tutte le risorse esistenti secondo lui sono sempre da riferirsi sia come stimolo che come processo di attuazione, allo sforzo che l'uomo per sua natura ha sempre effettuato a fini precisi innanzitutto di sopravvivenza poi del godimento umano, per il successivo perfezionamento umano ed infine quello dello spreco e della distruzione. In questi quattro cicli la componente primaria è sempre la componente economica che sostiene ogni concezione dell'idea dell'uomo che si concretizza nelle attività umane. Egli nota infatti come esse "si svolgono ritmicamente in una serie di impulsi"14 primari ed aggregati, minori e maggiori le cui ampiezze e frequenze sono non solo variabili, ma anche differenziate perché "mentre tutto è ritmico, non tutto è economico" 15 . Però la chiave di interpretazione della sua teoria è data dal fatto che pur se tali attività che scaturiscono dalle idee dell'uomo sia che si riconducano alla politica, alla guerra, alla filosofia oppure alla religione così come alla scienza o all'arte, al diritto ed alla medicina sono una trasformazione ritmica, vale a dire di cicli definiti, di potenzialità naturali che si realizzano per mezzo dello sforzo umano e anche se "nulla di ciò è in senso stretto, attività economica, eppure a condizionare tutte le culture, mescolandosi in maniera inestricabile con esse, vi è il fattore economico. I governi hanno i loro bilanci anche quando non li portano in pareggio; la religione e la legge devono avere le loro chiese e tribunali, i loro libri stampati e conservati, i loro ministri preparati; l'arte ha bisogno dei suoi materiali e delle sue gallerie, la scienza dei suoi laboratori, la medicina dei suoi ospedali, l'educazione della sua estesa gerarchia di scuole, collegi e università. Così la struttura materiale della dimora della cultura è economica, e il fondamento che sostiene questa sovrastruttura è il campo essenzialmente economico che riguarda il nutrimento, alloggio, vestiti, accessori, servizi e svaghi. Distinguiamo, perciò nel ritmo universale di ogni attività umana, una sovrastruttura pura di attività culturali, la struttura materiale di questa sovrastruttura e il fondamento di entrambe"16 Ecco allora come si perviene alla distinzione dei flussi sotto il simbolo $\mathrm{DA}^{17}$ che non rappresenta un flusso medio ma piuttosto un volume totale in cui rientrano tutti i flussi di attività umane che si inseriscono in un periodo di utilità di indefinita lunghezza in cui si attua una produzione, ma anche una distruzione o disintegrazione oppure si configura una scomparsa o una perdita di tutto ciò che rappresenta il presupposto economico di ogni realtà umana e che Lonergan riassume in "aggregato di pasti, vestiti, case, fattorie, miniere, strade, mercati, navi, città, industrie, servizi, svaghi, scuole, tribunali, parlamenti, ospedali, chiese."18 La cosa insolita del contenuto di questo simbolo è il suo significato di tasso quantitativo riferito a "tanto ogni tante volte" e non già "quanto ogni tante volte". ${ }^{9}$ Pertanto va

\footnotetext{
13 B.J. Lonergan, op. cit., pag. 43

14 Ibidem

15 Ibidem

16 B.J. Lonergan, op. cit., pag. 44

17 dove A sta per "attività economica" e D invece rappresenta la serie di eventi, il flusso di impulsi che caratterizza un ritmo composito costituito di molti ritmi di maggiore o minore grandezza variabilità e frequenza cfr. B.J. Lonergan, op. cit., pag. 45.

18 Ibidem

19 Ibidem; inoltre va precisato per una chiara comprensione che pur se trattasi di tasso quantitativo, non è comunque misurabile in quanto si riferisce a flussi temporali distinti però sotto il profilo qualitativo così
} 
considerato non come una quantità di un ritmo, di un volume o di un flusso totale, ma come un tasso quantitativo applicato a differenze qualitative come lo sono i colori in uno spettro. In egual maniera il simbolo DA non può essere considerato né un numero né un simbolo algebrico ordinario, anche se possiede analogie o parallelismi matematici. Per poter assegnare un'unità di misura in termini matematici occorre introdurre il concetto di moneta, che però ne indica soltanto la quantificazione, perché il valore, inteso nella sua realtà qualitativa, e quindi riferito allo sviluppo delle attività umane, non potrà in nessun modo quantificarsi matematicamente. ${ }^{20}$

Il simbolo DA allora si distingue in ritmi di base che a causa della accelerazione divengono poi distinti in una struttura dinamica di aggregato di flussi primari rappresentati con DA' e di aggregato di flussi secondari rappresentati con DA". Dice infatti Lonergan che stante il ritmo di base, di cui abbiamo parlato e rappresentato dal concetto di produzione "punto a punto" questa primaria struttura materiale diviene molto più significativa se la si considera invece sotto il suo profilo dinamico, rappresentato dai livelli differenti di produzione in serie: "le serie al livello inferiore hanno un volume di flusso proporzionato a quello dei prodotti finiti; ma le serie al livello successivo hanno un volume che è proporzionato all'accelerazione del livello inferiore; le serie al terzo livello hanno un volume proporzionato all'accelerazione dell'acceleratore del livello inferiore e così via."21

Ecco allora che dalla produzione "punto a punto" si passa a quello "punto sulla linea" ed in fine a quella di "punto sul piano". Per chiarire meglio Lonergan usa un esempio pratico: "Così, una tonnellata di ferro può essere impiegata in ciascuno dei tre livelli. Impiegata al livello inferiore, una tonnellata di ferro produce una tonnellata di parti di automobile o attrezzi agricoli. Impiegata al secondo livello una tonnellata di ferro produce una tonnellata di macchinari per fabbricare automobili, attrezzi agricoli o quello che si vuole. Impiegata la terzo livello, una tonnellata di ferro produce una tonnellata di macchinari per produrre i macchinari con i quali vengono prodotte le macchine per produrre automobili o attrezzi." 22 Ecco come si spiega il concetto di flusso, di accelerazione e di tasso quantitativo qualitativamente considerato. Infatti ciascun incremento in ciascuno dei diversi livelli produce un valore in termini di output qualitativamente diverso: considerato sotto l'aspetto punto a punto, pari ad un definito numero di parti; sotto l'aspetto punto a linea, corrispondente ad un determinato flusso di macchinari che produce un indeterminato flusso di prodotti; sotto l'aspetto punto sul piano, corrispondente ad un determinato flusso di attività che producono un indefinito numero di macchinari per una altrettanto indefinito numero di prodotti. "in ciascun caso la tonnellata contribuisce ad un dato flusso: al flusso di autovetture; al flusso di macchinari della fabbrica; al flusso di macchine utensili. ${ }^{23} \mathrm{Ma}$ i tre flussi sono correlati in

come dice Lonergan "come comunemente distinguiamo le differenze quantitative delle diverse lunghezze d'onda della luce mediante le differenze qualitative dello spettro."

20 Anche se oggi si cerca di farlo in termini anche scientifici, per quanto forzatamente, da quella branca di studiosi che, a mio avviso superando i limiti della disciplina nei suoi aspetti concettuali, studiano la cosiddetta economia della felicità, come se quest'ultima fosse uno stato di normalità durevole da raggiungere.

${ }^{21}$ B.J. Lonergan, op. cit., pag. 46

22 B.J. Lonergan, op. cit., pag. 47

${ }^{23}$ per la costruzione della fabbrica 
modo strano: uguali incrementi nei flussi differenti non implicano uguali differenze nel risultato finale; un incremento al livello inferiore produce un incremento proporzionato a quel livello, pochi altri automezzi per esempio; un incremento al secondo livello produce anch'esso un incremento proporzionato a quel livello; ma la sua implicazione è un incremento indefinito al livello inferiore; in maniera analoga, il terzo livello sta nella stessa relazione al secondo come il secondo al primo. Questa differenza è la differenza che c'è tra distanza e velocità, e tra velocità e accelerazione." ${ }^{24}$ Ecco come si spiega il "tanto ogni tante volte" perché qualsiasi configurazione di attività economica non rappresenta che un aggregato di operazioni ripetitive, ciascuna delle quali produce "tanto ogni tante volte" ecco perché ogni parte del ritmo di base deve essere considerato come una velocità. Quindi se si considerano i prodotti finali dei diversi ritmi emerge che alcuni sono correlati in modo proporzionato, mentre altri in modo sproporzionato. Infatti tutto dipende dalle attività che si pongono in atto, cosi si possono costruire treni per portare tanti passeggeri o merci all'anno, oppure si possono costruire tante ferrovie per far transitare treni che possono trasportare indefinite quantità di merci e persone per indefinite quantità di stazioni oppure indefinite quantità di Paesi. Comunque sia che si conduca una fattoria, disboschi il terreno per creare una fattoria o che si costruiscano treni o ferrovie, oppure si sfruttino pozzi di petrolio o si creino fabbriche per la produzione di scarpe si tratta sempre di una attività di natura ripetitiva e quindi di una velocità "tanto ogni tante volte". "Ma benché tutte siano velocità, la loro funzione non è identica. La funzione di alcune è di essere semplici velocità; la funzione di altre è di essere velocità che accelerano altre velocità." 25 Senza ulteriori approfondimenti il tutto può essere spiegato dalla distinzione tra DA' e DA" dicendo che quest'ultimo è l'accelerazione del primo in tre modi: estensivo, aumentando cioè il numero o la grandezza delle unità di produzione; intensivo e quindi aumentando l'efficienza delle unità di produzione esistenti; di mantenimento, vale a dire di mantenere i ritmi di accelerazione dei diversi flussi esistenti. Va comunque considerato che queste strutture dinamiche subiscono cambiamenti e trasformazioni nel tempo dovute sia all'interdipendenza tra gli uomini, che ha dato origine allo scambio e al commercio sia al fatto che ad ogni periodo si sono succedute idee nuove che hanno prevalso "sugli interessi investiti nelle vecchie idee" 26 e che per dare il massimo frutto si sono realizzate attraverso l'assunzione del rischio di impresa che però in relazione al loro tempo devono essere fortemente supportate da lampi di immaginazione creativa e da notevole capacità di adattamento. "E ogni idea, una volta che abbia portato il suo frutto, deve rassegnarsi a morire. Una nuova idea è nuova soltanto al suo primo apparire. Essa giunge all'uomo non come un possesso duraturo ma come una servitrice provvisoria; ha i suoi giorni gloriosi o tristi; vive per un periodo che è lungo o breve a seconda della sua generalità, ma può venire sostituita da ulteriori alternative e in ogni caso verrà trasformata, forse senza venire riconosciuta, da generalizzazioni più alte." 27 Le trasformazioni economiche si sono succedute in ogni settore e nulla vieta che in futuro potremmo assistere addirittura alla scomparsa del lavoro, dell'industria e del commercio, della trasformazione

${ }^{24}$ Ibidem
${ }_{25}$ B.J. Lonergan, op. cit., pag. 48
${ }^{26}$ B.J. Lonergan, op. cit., pag. 54
${ }^{27}$ B.J. Lonergan, op. cit., pag. 54 
dell'agricoltura in una superchimica e persino di superare il denaro e la finanza e "da far diventare la solidarietà economica un ricordo ed il potere sulla natura l'unica differenza tra un'alta civilizzazione e l'orticultura primitiva." 28

Pur se tutto ciò è molto verosimile, come i tempi della globalizzazione e di internet ci stanno dimostrando, ${ }^{29}$ al momento però non ci siamo ancora arrivati. Certo che per poter progredire saremo obbligati a trasformarci, ma sempre rispettando una condizione per cui la nostra società "Non deve orientare il suo sforzo principale al prodotto finale ordinario del tenore di vita, ma al prodotto finale generale degli strumenti culturali. Non deve vantarsi del suo sviluppo estensivo, di aggiungere industria a industria e di nutrire l'anima dell'uomo con un'abbondante richiesta di lavoro. Deve vantarsi del suo sviluppo intensivo, dello sviluppo intensivo puro che aumenta il tempo libero globale, che aumenta la piena libertà di molti e in maniera crescente quella di tutti per il campo delle attività culturali." 30 Ciò che la nuova idea di sviluppo economico deve portare dunque è innanzitutto una maggiore libertà che promuova l'avanzamento e l'intensificazione culturale affinché l'essere umano possa rientrare in possesso del giusto tempo libero a lui dovuto, per dedicarsi alle attività culturali del pensiero, dello spirito e delle arti, cosa che non esiste più da quando è stata data importanza essenziale al ritmo globale delle trasformazioni economiche.

Appare evidente quindi che quando Lonergan asserisce che "La realizzazione e la successiva applicazione di nuove idee pratiche non può avvenire senza la cooperazione umana", rivoluziona l'assunto deterministico dell'economia per legarlo invece all'intelligenza dell'uomo, che suddividendo i compiti produttivi, stabilendo le qualità e le quantità di produzioni di merci e ridistribuendone i proventi, si appropria dei meccanismi economici e li domina attraverso la propria intelligenza, ne determina il giusto impiego in base alle esigenze dettate da quella cultura umana la cui regola è impostata da un essere che ha raggiunto l'auto-appropriazione cioè la coscienza di essere soggetto. (Comprendere e Essere cap.V,13).

\section{Sistema produttivo di valore}

Ecco quindi come introduce, sulla base del bene d'ordine (ibidem X,1.1) una visione nuova di economia dello sviluppo integrale dell'uomo, visione in cui l'economia acquista così (nell'Insight) un significato ed una portata di scienza umana riferita all'economia politica quale attività dell'uomo tendente a conseguire migliori condizioni del tenore di vita non solo per se stesso ma anche per l'umanità intera.

Egli parte dalla correlazione, sempre riferita alle diverse attività di impresa, tra le grandezza di DA' e DA espressa dalle due note leggi dei rendimenti crescenti e decrescenti mettendo in evidenza che lo sfruttamento di una nuova idea in un dato settore genera alla sua introduzione dei rendimenti crescenti che con il passare del tempo tendono poi a diminuire divenendo decrescenti. Collegando quindi ritmi e flussi Lonergan dice che si possono ridefinire i rendimenti crescenti e decrescenti ancorandoli

${ }^{29}$ pensiamo alle nuove tecnologie finanziarie, ai nuovi sistemi di trasporto, allo smart-working ecc.

30 Ibidem
} 
all'accelerazione del ritmo economico universale, sicché si può facilmente immaginare che tutti i processi introdotti che incrementano le possibilità successive di ottenere ulteriore accelerazione producono rendimenti crescenti, mentre le procedure, tradizionali, invecchiate, obsolete che riducono tale possibilità producono naturalmente rendimenti decrescenti.

Ecco dunque come si introducono in ambito di produzione estensiva i rendimenti decrescenti: ogni ampliamento che aumenta la dimensione e il numero delle unità produttive esistenti genera di conseguenza la necessità di impegnare maggiori risorse per la manutenzione, la riparazione ed infine la sostituzione delle strutture esistenti. Mentre "L'intensificazione, l'aumento dell'efficienza, riduce la manutenzione, le riparazioni, le sostituzioni; abbassa, dunque, lo zero effettivo e così rende possibile una ulteriore accelerazione."31 Il percorso diviene pertanto virtuoso perché se invece di un ampliamento estensivo, attuato dalle imprese, che genera rendimenti decrescenti si genera uno sviluppo intensivo che migliori non già le condizioni di vita materiali, bensì quelle della struttura materiale della cultura ne consegue uno sviluppo culturale foriero di un'altra trasformazione della struttura dinamica. Se dunque detta intensificazione non si limita allo sviluppo di un successivo stadio di natura estensiva dell'ampliamento delle strutture economiche, essa deve necessariamente comportare un aumento del tempo libero a disposizione dell'essere umano. Va da sé che questo tempo libero può essere sprecato o restare inutilizzato, alla stessa stregua di qualsiasi altra risorsa, ma se invece viene impiegato correttamente per fini di natura umana, allora produce lo sviluppo culturale che da luogo ad una ulteriore e nuova trasformazione. Questo si spiega con il fatto che il maggior tempo dedicato alla cultura permette di generare migliori idee di progresso e di trasformazione della realtà. Infatti la progettualità non è altro che una illuminazione prodotta su un'idea che genera a sua volta un insieme di idee che genera un progetto e che a sua volta genera una trasformazione innovativa. Quindi la trasformazione e l'intensificazione derivano dalle nuove idee l'ampliamento ne rappresenta lo sfruttamento. Se c'è solo sviluppo estensivo si va verso una stagnazione economica, mentre se le idee innovative non vengono messe in pratica attuazione, si annulla il processo di verifica e correzione delle stesse, eliminando la possibilità che idee successive correggano, migliorino e sviluppino le precedenti. E' così che si verifica il ritmo universale di "tanto ogni tante volte" quale ritmo di trasformazioni successive seguite da sfruttamento o da successioni di sfruttamenti che daranno luogo a una nuova trasformazione e quindi ad un nuovo inizio di maggiori potenzialità.

Concludendo si riassumono di seguito le fasi cicliche, indicate da B. Lonergan, del ritmo universale che la stratificazione dei settori imprenditoriali imprimono e che si susseguono come cicli di produzione di valore dando un risultato concatenato delle variabili DA' e DA":

1) "fase statica" in cui i due termini sono costanti con DA" allo stadio di zero effettivo;

2) "fase capitalista" in cui DA" è crescente con DA' costante;

3) "fase materialista" con DA" costante sopra lo zero effettivo che destina il suo surplus di produzione all'aumento del DA' ordinario ${ }^{32}$;

31 B.J. Lonergan, op. cit., pag. 57

32 Per DA' ordinario si intende cibo, vestiario, alloggio, comodità servizi, divertimenti 
4) "fase culturale" con DA" costante sopra lo zero effettivo che destina il suo surplus di produzione all'aumento del DA' generale. ${ }^{33}$

Come si può osservare le combinazioni differenti di DA' e DA" costanti o crescenti determinano nei 4 cicli descritti una fase capitalista che trasforma i mezzi di produzione; una fase materialista che sfrutta nuove idee per elevare il tenore di vita; una fase culturale che destina il benessere e il potere material a dotare di mezzi lo sviluppo di attività culturali; infine "una fase statica in cui il processo giace improduttivo e le attività non economiche si sviluppano indipendentemente dalle condizioni materiali."34

\section{Economia come bene d'ordine}

Da questa visione si determina quindi la nuova concezione rivoluzionaria dell'economia dello sviluppo che si focalizza su un paradigma diverso e costitutivo del punto centrale dello sviluppo che si riscontra proprio nell'impresa. Non già nell'azienda o meglio nell'organizzazione aziendale, quanto più invece nell'impresa, come idea imprenditoriale dell'essere umano che si assume il rischio di impresa e quindi di economia dello sviluppo. Per Lonergan quindi l'impresa si costituisce come bene d'ordine $^{35}$ e nella stratificazione dei ritmi deve essere rivolta al sostegno e alla salvaguardia della dignità dell'uomo ed alla promozione del bene comune, perché come bene d'ordine è un flusso di azioni e di benefici, fatte da un insieme di persone per un fine umano e pertanto tali flussi divengono cooperazioni i cui benefici non restano appannaggio del singolo individuo oppure del gruppo di uomini coinvolti che li hanno prodotti, ma vengono diffusi per il bene comune di tutta l'umanità. Si deve intendere, con ciò, un sistema che si rivela possibile attraverso un'analisi funzionale di tipo macroeconomico. Ma la questione è in ultima analisi esistenziale, perché si tratta di scegliere tra praxis e tecnica. Questa nuova visione economica attiene ad una nuova concezione del valore non tanto od esclusivamente economico-finanziario, ma quanto più elemento di misura esistenziale dell'impatto sociale del progresso, tecnologico, scientifico e multimediale sintetizzato nella conoscenza, che solo l'intelligenza umana proporzionata alla coscienza può individuare nel passaggio obbligato dalla conversione intellettuale a quella morale e poi da quest'ultima a quella religiosa. Solo così si concretizza il fine ultimo dell'uomo come elemento di conoscenza universale e quindi di testimonianza vera e completa di valore nell'obiettivo di divenire elemento prezioso di sviluppo della conoscenza che crea il valore economico propriamente inteso. L'economia dunque si realizza nei ritmi e nei flussi creatori di valore che si concretizzano nell'impresa che scaturisce da un'idea dell'essere umano e per la quale non conta il tradizionale trade off tra to buy or to make, ma la priorità dell'inserimento dell'essere umano, nei ritmi e redistribuzione di valore. ${ }^{36}$

\section{Scuola di una rinnovata economia dello Sviluppo}

33 Per DA' generale si intende la struttura materiale della cultura, gli strumenti del sapere e delle professioni.

${ }^{34}$ B.J. Lonergan, op. cit., pag. 63; da cui naturalmente ripartono gli altri tre cicli.

35 il concetto di bene si misura su tre livelli: 1 . il bene d'appetito; 2 . il bene d'ordine; 3 . il bene valore.

36 ciò che contano sono le potenzialità e gli skill oltre che le conoscenze delle persone messe al servizio dello sviluppo anzi del bene comune, come recita il punto 15 dell'enciclica Populorum Progressio. 
Partendo quindi dalla concezione innovativa dell'impresa come bene d'ordine che scaturisce dalla conoscenza fondazionale dell'essere umano auto-proporzionato abbiamo proposto un'esperienza che vorremmo fare sulla riscoperta di questo pensiero attraverso la creazione di una Scuola di Pensiero di Economia dello Sviluppo Integrale dell'Uomo. Un'idea di Scuola rinnovata perché fondata sul bene d'ordine dell'impresa da cui deve ripartire la nozione di sviluppo integrale dell'uomo, come auto-sviluppo, (Comprendere e Essere IV,4) come messa a frutto dei suoi talenti perché solo dall'impresa così concepita può venire un vero sviluppo, perché dice Lonergan nel suo Essay: "In definitiva io direi che il problema della libera impresa è a prima vista scientifico, ma in ultima analisi esistenziale. E' in prima approssimazione scientifico in quanto non si deve intendere per libera impresa il sistema che è andato avanti in occidente negli ultimi duecento anni. E' esistenziale perché coinvolge non tanto i mezzi disponibili più o meno scarsi che si trovano in natura, quanto più le conoscenze che creano l'insight innovativo che assumendosi consapevolmente il rischio di impresa è intenzionalmente rivolto al bene comune e dunque al bene d'ordine.

Ecco allora delinearsi un percorso che la Scuola di Economia dello Sviluppo Integrale di Kinshasa (RDC) promuoverà nel mondo intero, in tutti i paesi, a prescindere dal loro livello di sviluppo meramente economico, trovando nuovi elementi di valutazione quali la revisione esistenziale delle teorie dello sviluppo del secolo scorso per porle più ordinatamente in una più corretta visione di teorie della crescita economica.

La scuola è stata fondata a Kinshasa (RDC) il 17 aprile del 2013 presso l'Università Cattolica del Congo.

\section{Sviluppo come altro nome della pace}

Questo nuovo concetto di economia dello sviluppo risponde ai principi della Dottrina Sociale della Chiesa e anche se contempla quella branca dell'economia che applica le moderne tecniche di analisi matematico-statistiche della scienza macroeconomica e microeconomica di sviluppo socio- economico, ambientale e istituzionale rivolte ai cosiddetti Paesi in Via di Sviluppo, riteniamo che questo tipo di economia debba manifestare discontinuità dalle teorie e dalle visioni del secolo scorso. Questa nuova visione non può più accettare il capitalismo-liberista come unica base per la crescita economica e come vettore dello sviluppo promosso dall'investimento più opportuno mirato al profitto, al risparmio ed alla produttività industriale. Questa nuova idea di economia dello sviluppo che abbiamo in programma di promuovere deve mirare principalmente alla dignità dell'uomo ed al suo sviluppo integrale. Essa deve affrontare le determinanti della povertà e del sottosviluppo nonché delineare le politiche da attuare per ottenere che i Paesi in via di sviluppo escano non solo dal loro sottosviluppo, ma anche soprattutto dall'ignoranza. Appare inconfutabile che il sottosviluppo e la povertà dipendano dal grado attuale di ignoranza esistente nella gente dei paesi che chiamiamo poveri. La mancanza di conoscenza, soprattutto in campo socio-economico consente lo sfruttamento selvaggio e irrazionale non solo delle risorse materiali, ma anche delle risorse umane che sono spesso prive di protezione e di rispetto per la loro dignità a causa di abuso di poteri da considerarsi ancora di tipo colonialista da parte delle multinazionali. Ciò che la classifica dei Paesi poveri dimostra, nonostante gli enormi flussi di denaro loro trasferiti nel tempo, è che lo sviluppo non può venire solo dal trasferimento di 
ricchezza, ma dall'efficace trasferimento di conoscenze ai poveri che si confrontano con i problemi del sotto-sviluppo. E' inutile dare macchine a persone che non sanno servirsene, occorre invece insegnare loro come produrle direttamente al fine del loro migliore utilizzo. Per questo è molto importante identificare gruppi sociali e attori capaci di costituire la base socio-politica di supporto per strategie di sviluppo sostenibile nei vari livelli della collettività. L'economia di mercato senza la partecipazione attiva di uomini di buona volontà, non permetterebbe uno sviluppo sostenibile, perché tenderebbe a sfruttare la conoscenza per i gruppi che hanno il potere e che praticano il capitalismo di sottrazione. Una nozione nuova dello sviluppo integrale visto come integrativo, trasparente e completo, come rifiuto del liberismo economico, un concetto di sviluppo a cui Lonergan ha sempre mirato e che è stato definito dall'enciclica Populorum Progressio (76) il "nuovo nome della pace". Per lui dunque l'economia è un fatto esistenziale è un fatto di conoscenza e con ciò ingenera in tutti gli studiosi la convinzione che non è il mero scambio o il trasferimento di ricchezza a creare sviluppo bensì il trasferimento di conoscenza. In tale ambito allora sarà importante, nella nozione esistenziale di economia rivolta allo sviluppo, definire i caratteri che quest'ultimo deve assumere come elementi portanti; deve essere cioè sostenibile equilibrato e durevole.

\section{Economia come conoscenza}

Tutto ciò per sottolineare che la conoscenza è ricchezza. E' quindi necessario lottare per sostenere una economia rivolta alla diffusione delle conoscenze, al sostegno di quei cambiamenti fondamentali che permettano di aumentare e accumulare la ricchezza materiale necessaria. Così, le barriere sistemiche saranno superate al fine di raggiungere un equilibrio tra le esigenze di sviluppo integrale (sostenibile, durevole, equo) e quelle delle forme dominanti del capitalismo di sottrazione. La conoscenza è sempre stata una risorsa importante per la produzione di un'impresa (che è differente dalla produzione naturale o animale perché è caratterizzata dall'uso nell'opera di capacità intellettuali), ma oggi è diventata ancor più forza produttiva fondamentale che si basa sulla conoscenza scientifica e le sue opportunità di sviluppo come conoscenza indipendente e non più fondata sul potere religioso o politico. La conoscenza, che ha realizzato la conquista della propria autonomia attraverso un processo di verifica della verità, si chiama sperimentazione. Vale a dire, la riproducibilità di quanto si afferma in termini di relazioni causa-effetto. Ecco quindi il percorso di emancipazione dell'uomo: una volta che si acquisisce la conoscenza che ha caratteristiche di riproducibilità, l'economia reale ha a sua disposizione una grande risorsa che consiste in una conoscenza astratta (della scienza e della tecnologia) costruita in modo da essere riproducibile dieci, cento, mille volte e in ambienti diversi. Così si afferma il capitalismo delle macchine utensili, che nasce dalla rivoluzione industriale; il capitalismo meccanizzato della catena di montaggio e dell'automazione, nato dalla rivoluzione industriale iniziata nella metà del XVIII secolo come moderna economia si sviluppa principalmente attraverso la tecnologia delle macchine utensili che incorporando conoscenza riproducibile su base scientifica, consente economie di scala enormi.

Così si sviluppa infine e si afferma il capitalismo delle macchine digitali e della robotica che a differenza dei processi meccanici permette attraverso sofisticati algoritmi di 
produrre in assenza umana, innovazione creativa ed operatività in termini scientifici, più produttive. Infatti, la ricerca e l'assemblaggio del processo meccanico utilizzati per progettare il prototipo originale possono essere riutilizzati a costo zero per costruire la seconda, decima, la millesima macchina. E il processo lavorativo impiegato per progettare la prima unità di prodotto ottenuto dalle macchine utensili può essere riutilizzato più volte per migliaia o milioni di prodotti identici. E il processo di innovazione digitale permette di replicare infinitamente anche a distanza ed in delocalizzazione fisica, milioni di processi identici che creano oggetti identici prodotti da sistemi robottizzati fatti sempre più a configurazione umanoide. Si capisce così che è la produttività che crea valore e, pertanto, anche le ipotesi di crescita economica per sostenere lo sviluppo assumono nuove forme e configurazioni evolutive. La produzione efficiente che dà la possibilità di ottenere più profitto si basa sulla conoscenza e sul miglioramento continuo dei sistemi di produzione. Si tratta come detto da B.J. Lonergan di uno spostamento significativo del sistema dal processo di produzione punto a punto, in cui il valore corrispondente ai prodotti è strettamente rapportato ai materiali impiegati, senza possibilità, se non c'è conoscenza, di passare ad un sistema di punti sulla linea in cui la creazione di valore è correlata alla quantità illimitata di prodotti che una macchina utensile permette di produrre durante tutto il suo ciclo di vita, per diventare finalmente un sistema di punto sul piano, dove non c'è più corrispondenza tra i materiali utilizzati la macchina utensile adoperata e i prodotti ottenuti, a prescindere dall'implicazione umana, in quanto il processo è quello di fornire servizi le cui possibilità di produzione sono infinite, sia in termini di quantità che in termini di possibilità illimitate del servizio erogato. Questo significa che ogni euro (o ora di lavoro), di nuove conoscenze investito nella produzione può rendere di più o molto di più, dal momento che questa conoscenza può essere utile - generando valore per gli utenti - non una, ma dieci, cento, mille, milioni di volte, con effetti moltiplicatori che cambiano radicalmente il senso della produzione rispetto all'economia pre-industriale, e post industriale. L' innovazione chiave della modernità è che diventa conveniente investire nei processi di apprendimento proprio grazie alla natura riproducibile della conoscenza, è che diventa certezza di accumulazione continua di conoscenza riproducibile grazie a processi digitali sempre più sofisticati e dematerializzati. Ecco perché non si lavora più per ripetere le medesime e tipiche operazioni, come nell'artigianato, bensì per innovare, inventando nuove macchine, nuovi prodotti, nuove soluzioni, nuovi processi. Nuovi hardware e nuovi software in un mondo sempre più digitale e meno umano. In passato la conoscenza veniva certamente utilizzata, ma non essendo conoscenza riproducibile, la portata limitata del suo utilizzo, non rendeva conveniente l'investimento nei processi di ricerca e sviluppo. Così come l'acqua è fonte di vita la conoscenza è la fonte primaria dello sviluppo integrale. Infatti, come l'acqua permette la nascita della vegetazione nel deserto, la conoscenza crea ricchezza reale per l'uomo e cioè il suo sviluppo. Tuttavia, questa conoscenza deve essere ben guidata affinché possa davvero dare risultati positivi per la comunità degli uomini nella prospettiva dello sviluppo integrale. 


\section{I vettori dello Sviluppo}

Ciò che la Scuola si prefigge di introdurre con il suo avvio è stato dichiarato in un manifesto e questo manifesto è una nuova concezione di capitalismo definito "Neocapitalismo etico" che in discontinuità con il neoliberismo - si caratterizza per il trasferimento della conoscenza alle persone povere dei paesi in via di sviluppo sul loro territorio principalmente attraverso la formazione della classe dirigente la quale dopo aver appreso la conoscenza delle tecniche di ricerca e i diversi modelli di sviluppo possa applicarli, filtrandoli con la propria cultura, tenendo conto dell'ambito intellettuale in cui vivono e nel rispetto delle tradizioni e la storia del loro popolo. Ritenendo dunque che ogni persona abbia sue specifiche doti e potenzialità naturali il vettore dello sviluppo è lo "sfruttamento della materia grigia" 37 vale a dire delle attitudini e delle potenzialità esistenti sin dalla nascita nel loro DNA e nel loro cervello. Essendo diverse per ciascuna persona determinano anche il quadro di riferimento del loro impiego. Così l'impresa come bene d'ordine ricercherà per le sue necessità di creazione sviluppo e crescita tutte le potenzialità umane esistenti sul territorio in funzione dell'ottenimento del bene comune individuato non più semplicemente in ragione di un tradizionale "business plan", quanto più in un piano concreto di progettualità mirata allo sviluppo che sappia misurare le necessità umane dell'impresa non più fondata sull'innovazione tecnologica orientata prioritariamente al profitto, ma soprattutto sulla "innovativa tecnologia mentale di sviluppo" indirizzata al bene comune. I vettori fondanti dunque consistono nella formazione di esatte "conoscenze scientifiche"; nella acquisizione di nuovi "modelli di organizzazione" socio-economica; di "puntualità e precisione di processi” basati sulla coscienza del rispetto delle loro regole fondative; infine sulla "capacità di autodeterminazione" che aspiri sempre al risultato in termini di dignità, sia con criteri di efficacia che di efficienza. L'impresa intesa in questo senso vede dunque innanzitutto la formazione di una coscienza della dignità professionale individuale; l'impiego delle potenzialità umane insite in ogni personalità unica ed irripetibile; l'ampliamento della visione produttiva in termini di creazione di valore per l'impresa e non già del tradizionale valore per gli azionisti; il tutto a sostegno di una entità di bene d'ordine che persegue una giustizia sociale nell'ambito di organizzazioni in cui ci sia edificazione delle attitudini e potenzialità, e dunque si compia sempre lo sforzo sociale ed economico di mettere l"'uomo giusto al posto giusto". Infatti, solo la profonda motivazione di ciascuno a svolgere con amore la propria attività può produrre cooperazioni foriere di benefici per tutti.

\section{Indice di riferimento dello sviluppo: sostituire il PIL con l'IPI}

Partendo dall'impresa come punto di riferimento di produzione, trasformazione, distribuzione e consumo, e dai ritmi esistenti nei cicli sopra evidenziati dobbiamo aggregarne $\mathrm{i}$ flussi sia in termini micro che macro-economici onde arrivare ad una possibile e coerente misurazione. La misurazione può essere tanto della crescita

37 Di ciò ha parlato in una sua relazione il Cardinale Laurent Monsengwo Pasinyia nella sua relazione sullo sviluppo, tenuta presso l'Accademia delle Scienze in Vaticano nel 2015. 
economica che di quella sociale, che di quella ecologica. Questo ultimo termine collegato al primo permette di definire quella sintesi che si chiama economia dello sviluppo integrale. Oggi siamo ancora in tempo per riprendere il discorso economico in termini umani e non più numerici o econometrici o ancor peggio digitali. La Scuola di Pensiero di Kinshasa propone un altro tipo di misurazione, più umana rispetto a ciò che misuriamo con il PIL ${ }^{38}$. Una misura che non rappresenti solo ciò che il Paese produce, ma una misura che indichi in maniera chiara lo stato di sviluppo di un Paese basato sulle proprie potenzialità. Ecco dunque che la misura dello sviluppo dei Paesi non sarà più il P.I.L. ma l'I.P.I. (Indice di Potenzialità Inutilizzata) nei suoi caratteri umani, animali e ambientali. ${ }^{39}$ A questo misuratore se ne deve aggiungere un altro determinato dalla necessità che qualsiasi investimento abbia il dovuto ritorno sociale e pertanto deve avere un misuratore di Impact Finance, vale a dire che ogni investimento, nei settori citati, non deve essere valutato soltanto in base al suo ritorno economico-finanziario, bensì dall'impatto sociale del suo investimento e cioè dalla proporzione esistente tra il ritorno economico ed il ritorno sociale adeguatamente ponderato in termini di sviluppo integrale nelle sue diverse caratteristiche: se tale rapporto è superiore alla percentuale di (S) nel Paese allora l'investimento tendente allo sviluppo ha ragione di essere effettuato. ${ }^{40}$

\section{Conclusione}

La conclusione a cui il presente studio della nuova visione economica di B.J. Lonergan, permette di giungere, che ritengo essere non solo un punto di arrivo, ma anche di partenza per tutti gli studiosi della disciplina, è che l'economia è un fatto esclusivamente umano rispondente a regole che non possono essere distinte dalla consapevolezza che $\mathrm{i}$ suoi strumenti devono essere rivolti al bene comune e non all'arricchimento individuale di stampo capitalistico liberista. In tale contesto lo strumento economico per antonomasia è l'impresa, ma non in senso tradizionale, bensì impresa come bene d'ordine, rivolta alla produzione di benefici per tutto il contesto che

\footnotetext{
38 è l'acronimo di Prodotto Interno Lordo ed è in parole molto semplici la ricchezza che un certo Paese è in grado di produrre nell'arco temporale di un anno. Si tratta della somma totale dei beni e dei servizi che si producono per essere consumati all'interno di uno Stato

${ }^{39}$ Poiché la produzione di ricchezza non dimostra lo sviluppo, per ottenere l'indicazione occorre partire dalle potenzialità esistenti nel Paese e quantificarne l'impiego produttivo nel periodo stabilito, vale a dire nell'anno onde comprendere quanta parte di esse non viene utilizzata. L'Indice di Potenzialità Inutilizzata (IPI) la cui diminuzione indica la percentuale di Sviluppo $(S)$ che si è raggiunto nel perodo, dipende quindi dalla somma delle differenze scientificamente rilevate tra Potenzialità Fisiche esistenti ( $\mathrm{PFe}$ ) e Potenzialità Fisiche impiegate (PFi); tra Potenzialità Animali esistenti (PAe) e Potenzialità Animali impiegate (PAi); tra Potenzialità Umane esistenti $(\mathrm{PUe})$ e Potenzialità Umane impiegate $(\mathrm{PUi})$ e cioè $\mathrm{IPI}=(\mathrm{PFe}-\mathrm{PFi})+(\mathrm{PAe}-$ PAi) $+($ PUe-PUi) da cui (IPIt $2 /$ IPIt 1$)=\delta S$.

${ }^{40}$ ponendo $\mathrm{S}$ come percentuale di sviluppo realizzato nel Paese a livello di settore valutato (F,A,U)e per il periodo di osservazione, un investimento produttivo dovrà esprimere oltre al ritorno sul Capitale investito (ROI) anche un ritorno in termini sociali valutato sulla base della percentuale di sviluppo derivante dall'impiego di maggiori potenzialità. Naturalmente se questo ritorno è superiore alla percentuale di $\mathrm{S}$ l'investimento ha anche una validità sociale. L'approfondimento dell'impatto sui diversi ritmi economici è ancora tutto da studiare.
} 
vi prende parte in termini di rischio-esposti. ${ }^{41}$ Per l'elaborazione dell'Indice IPI e l'attuazione di questa nuova visione di economia dello sviluppo si fa riferimento al manifesto della Scuola di Pensiero di Economia dello sviluppo integrale dell'Uomo creato a Kinshasa ${ }^{42}$ aperto al contributo di ogni ricercatore. La visione economica di B.J. Lonergan, resta un fondamento innovativo su cui rifondare il pensiero di chi vuole riscoprire il valore della dignità dell'uomo che si misura dal grado di libertà e di capacità lavorativa in termini di creatività, dell'essere umano. I ritmi così come concepiti dovrebbero finalmente condurre ad una società fondata sullo sviluppo equo, sostenibile e durevole, il cui obiettivo è quello della crescita umana e non dell'arricchimento economico in una frenetica accumulazione che avviene sempre a scapito di qualcun altro o come sfruttamento come strumentalizzazione o come esclusione. Lonergan invece attraverso il bene d'ordine teorizza un'impresa che sapendo interpretare i ritmi, sappia creare e ridistribuire valore nell'ambito di una giustizia sociale propriamente intesa, dove ogni essere umano possa sentirsi incluso e soggetto di un bene comune come deve essere: il bene di tutti e di ciascuno inteso come obiettivo di sviluppo integrale vale a dire integrativo, trasparente e completo.

\section{Bibliografia}

B.J. Lonergan For a New Political Economy, ed. By Ph.J. McShane (CWBL 21), University of Toronto Press, Toronto 1998 - Traduz. Ital. di Michele Tomasi Studi di Economia Città Nuova Editrice, Roma Novembre 2013

B.J. Lonergan Macroeconomics Dynamics: An essay on Circulation Analysis (Collected Works of Bernard Lonergan, Volume 15). Edited by F.G. Lawrence, P. H. Byrne, and C.C. Hefling, Jr.. Toronto: University of Toronto Press, 1999.

B.J. Lonergan INSIGHT A Study of Human Understanding Collected works of Bernard Lonergan - vol. 3 PUBLISHED FOR Lonergan Research Institute of Regis College, Toronto 1992. Per la Versione italiana INSIGHT Uno studio del comprendere umano a cura di Saturnino Muratore, Natalino Spaccapelo 2007, Citta Nuova Editrice, Roma.

B.J. Lonergan Understanding and being - The Halifax lectures on Insight - Collected works of Bernard Lonergan vol. 5 PUBLISHED FOR Lonergan Research Institute of Regis College, Toronto 1990. Per la Versione italiana COMPRENDERE ED ESSERE Le Lezioni di Halifax su Insight Edizione italiana a cura di Saturnino Muratore, Natalino Spaccapelo 1993, Citta Nuova Editrice, Roma.

Palo VI, Enciclica Populorum Progressio 1967 ed. Paoline

Joseph A. Schumpeter Capitalismo, socialismo e democrazia Etas editore 2001,

Joseph A. Schumpeter Teoria dello sviluppo economico, Etas editore 2002

Joseph A. Schumpeter Storia dell'analisi economica dal 1870 a Keynes - vol.3, Bollati Boringhieri, 2^ ed. 1990

Kondratieff Waves in the World System Perspective. Kondratieff Waves. Dimensions and Perspectives at the Dawn of the 21st Century / Ed. by Leonid E. Grinin, Tessaleno C. Devezas, and Andrey V. Korotayev. Volgograd: Uchitel, 2012. P. 23-64.

Alexander Aivazov and Andrey Kobyakov Nikolai Kondratiev's "Long Wave": The Mirror of the Global Economic Crisis Global Research, November 27, 2008 (www.globalresearch.ca) Versione Italiana La teoria dei cicli di Kondratiev specchio della crisi economica globale (http://www.ariannaeditrice.it/articolo.php?id articolo=22834) di Alexander Aivazov e Andrej Kobyakov - 09/12/2008

41 tutti i soggetti conosciuti con il termine inglese di stakeholder; cfr. Romeo Ciminello Il significato cristiano della responsabilità sociale dell'impresa, Tipar editrice Roma 2008

42 cfr.: http://agenda-etica.blogspot.it/search?q=manifesto + di + economia + dello + sviluppo + integrale + dell $\% 27$ uomo 
Kondratieff Waves in the World System Perspective. Kondratieff Waves. Dimensions and Perspectives at the Dawn of the 21st Century / Ed. by Leonid E. Grinin, Tessaleno C. Devezas, and Andrey V. Korotayev. Volgograd: Uchitel, 2012. P. 23-64

Juglar, Clément (1819-1905). Des crises commerciales et de leur retour périodique en France, en Angleterre et aux États-Unis / par le Dr Clément Juglar.... 1862. http://gallica.bnf.fr/ark:/12148/bpt6k1060720

Viviane Forrester L'orrore economico, Edizioni TEA Saggi 1999

L. Monsengwo,(29/11/2013) Relazione sullo sviluppo integrale solidale e durevole in Africa https://ilsismografo.blogspot.com/2013/11/30nov-ossrom-africa-sviluppo-solidale-accademiascienze.html?m $=0$

R. Ciminello (7-8/10/2014) Mutualita': cambio di prospettiva il salto di paradigma nella responsabilita' sociale dell'impresa http://www.academia.edu/29359452/MUTUALITA_PAPER_RC

R. Ciminello Il significato Cristiano della Responsabilità sociale dell'impresa, Tipar Editrice Roma 2008 